\title{
COMPLÉXITES ÉDUCATIVES ET PEDAGOGIQUES
}

\author{
Auteur : \\ Jean-Marc FERT \\ Éducateur \& formateur \\ Lycée Henri-IV, Paris \\ jmfert@yahoo.fr
}

\section{Résumé :}

Éduquer est la tâche créative par excellence puisqu'il s'agit de faire émerger une personne humaine libre, socialisée et auteure de sa vie à partir d'un homo sapiens nouveau-né, l'un des animaux les plus faibles et les plus dépendants à la naissance.

Cette tâche était vue comme l'une des trois tâches impossible par Freud qui ne disposait pas encore des outils théoriques permettant de la penser. Pensée systémique temporalisée, cybernétique du second ordre de Von Foerster et pensée complexe morinienne sont requises pour comprendre l'éducation.

Les crises actuelles de l'éducation, l'indigence des propositions politiques dans ce domaine, l'inertie et la dégradation du système éducatif, tout cela est compréhensible lorsque l'on constate le terrible manque d'une approche systémique et anthropologique des relations et situations éducatives.

Une simple systémographie du système éducatif ne suffit pas. L'espoir ne peut renaître qu'en considérant l'évolution du petit d'homme dans sa complexité chrono-bio-socio-psycho-auto-anthropo-logique, au sein de son milieu de vie lui-même d'une complexité multidimensionnelle.

Il nous faut partir des reliances éduquantes et d'une interprétation systémique des pédagogies "cré-actives" pour comprendre les mutations efficientes de l'éducation et des systèmes éducatifs.

\section{Mots-clés :}

Éducation, pédagogie, complexité, jeunesse, mutation, phylogenèse, résistance, reliance, créativité, projet.

\section{INTRODUCTION}

Je commence à écrire ces lignes encore attristé par la disparition d'André de Peretti. Éducateur, formateur, penseur de l'éducation et de la formation, il avait été en 1982 l'auteur du fameux rapport sur la formation des personnels de l'Éducation Nationale. J'en cite ici brièvement un passage, où il décrit les bases de la formation théorique des personnels de l'éducation: «Il est opportun d'y ajouter des possibilités de conceptualisation transdisciplinaire majeures (analyse systémique, théorie des modèles, théorie des recherches en sciences humaines, etc.).» ${ }^{1}$.

L'exigence, la précision et l'éthique humaniste de sa pensée nous placent d'emblée au cœur de notre sujet. Reprenons.

Quelle place pour l'humain demain, alors que l'ouvrier est remplacé par le robot et le décideur par l'algorithme? «L'homme du futur», nous dit-on, «se réalisera par son inventivité et sa créativité, accompagné de systèmes automatisés qui l'auront libéré des tâches répétitives. »

Le plus systémicien ${ }^{2}$ de nos grands hommes politiques, Michel Rocard, avait bien compris dès 1988 l'une des conséquences de ce constat. Il avait mis l'éducation comme première priorité de son action. En effet, sans l'éducation, le petit d'homme n'est qu'un primate sans défense naturelle et peu viable. C'est l'éducation qui peut en faire un être social créatif. L'éducation est donc la fonction la plus créative que l'on puisse imaginer, puisqu'il lui incombe de créer les créateurs de demain.

\footnotetext{
${ }^{1}$ De Peretti, A. (1982). La Formation des personnels de l'Éducation nationale, p. 112. La documentation française, Paris.
} ${ }^{2}$ Membre du fameux « groupe des dix », qui comprenait notamment Henri Atlan, Joël de Rosnay, Edgar Morin.. 
Soucieux de la formation des éducateurs, André de Peretti voit donc dès 1982 qu'il leur faudra «penser système » pour espérer mener cette tâche à bien.

Puis, c'est Edgar Morin qui passe du «penser système » à la pensée complexe, et qui met lui aussi la réflexion sur l'éducation au cœur de son impressionnante activité. Dans l'un de ses derniers ouvrages, il nous propose de changer l'éducation pour qu'elle puisse «Enseigner à vivre $»^{3}$ à la jeunesse de «Demain ». Si le mot demain est ici entouré de guillemets, c'est bien entendu en référence au film à succès de Mélanie Laurent et Cyril Dion. En effet l'éducation est confrontée aujourd'hui au défi de préparer la jeunesse à un monde à l'évolution totalement imprévisible, en métamorphose radicale, en proie à un ensemble de crises systémiques dont l'enjeu semble de plus en plus être l'existence même de l'espèce humaine, menacée par des dégradations irréversibles de ses milieux de vie biologiques, sociaux, politiques, religieux, et culturels. La pensée complexe morinienne nous permet de voir clairement l'intrication, les interrétroactions entre les différentes facettes d'une multicrise globale de notre modèle de développement. La créativité de la nouvelle génération devra nécessairement s'orienter vers l'invention de sorties de crise les moins catastrophiques possibles.

Bien entendu, ce court article ne sera qu'une esquisse. Il s'agit ici de montrer en quoi l'approche systémique et le penser complexe sont féconds pour concevoir ces nécessaires métamorphoses des institutions et pratiques éducatives. Je commencerai par rappeler pour quelles raisons j'utilise plus volontiers le terme «penser complexe » que le terme «approche systémique ». Nous verrons ensuite pourquoi, en matière d'éducation, la critique du rationalisme cartésien entraine certains courants vers un abandon total d'une pensée rationnelle, et comment le penser complexe permet d'échapper à ces nouveaux obscurantismes. Puis nous montrerons en quoi un entrainement à l'usage des outils systémiques nous donne une meilleure capacité d'action et d'innovation dans un monde en mutation constante. Enfin au cœur de notre sujet, une approche complexe des différentes dimensions des pratiques éducatives nous permettra de comprendre la cohérence des facteurs qui favorisent l'émergence des capabilités d'évolution, de créativité et d'innovation.

\section{APPROCHES SYSTÉMIQUES OU PENSÉE COMPLEXE}

Depuis mes premiers travaux, j'ai utilisé des approches systémiques des situations et pratiques éducatives. En reprenant le texte de Bateson ${ }^{4}$, il était facile de montrer la « schismogénèse » à l'œuvre dans la multiplication des relations complémentaires entre maître et élève dans la plus classique des pédagogies ${ }^{5}$. C'est « "'inflation complémentaire » que Ionesco a mis en scène de façon parodique dans «La Leçon». Ce sont aussi des approches systémiques qui ont mis en lumière « La Crise de l'organisation scolaire ${ }^{6}$.

Cependant, j'ai compris que le «monde» systémique connaissait une petite révolution lorsque j'ai participé au fameux colloque de La Villette en octobre 1990 et que j'ai pu y entendre le splendide exposé de Heinz Von Foerster sur la cybernétique du second ordre ${ }^{7}$. Il y montrait avec toute sa rigueur logique la nécessité de l'implication du systémographieur dans son analyse de système, du thérapeute dans son intervention en thérapie familiale systémique, ou de l'éducateur dans sa compréhension de l'élève et son intervention auprès de lui. Pour moi, la systémique rencontrait enfin l'intimité de mon implication personnelle d'éducateur, héritée de mes origines rogeriennes.

\footnotetext{
${ }^{3}$ Morin, E. (2014). Enseigner à vivre. Acte Sud, Arles.

${ }^{4}$ Bateson, G. (1972, trad. fr. 1977). Vers une écologie de l'esprit 1, pp. 77-87 \& 103-119. Seuil, Paris.

${ }^{5}$ Fert, J.-M. (1998). La Professionnalisation des conseillers principaux d'éducation, pp. 74-76. L'Harmattan, Paris.

${ }^{6}$ Obin, J.-P. (1993). La Crise de l'organisation scolaire. Hachette, Paris.

${ }^{7}$ Von Foerster, H. (1991). «Éthique et cybernétique de second ordre», in Rey Y. \& Prieur B. (Sous la dir. de), Systèmes éthique perspectives en thérapie familiale. E.S.F., Paris 
Ma seconde révolution eut lieu par ma lecture et ma compréhension des travaux du prix Nobel Ilya Prigogine. Il a montré que dans des circonstances finalement fort fréquentes dans la nature, la matière la plus simple et la plus élémentaire (un peu d'eau, par exemple) peut adopter des comportements complexes ${ }^{8}$, et semble agir comme à la recherche d'un but. Il insiste également sur l'irréversibilité du temps.

Pour finir, la pensée complexe d'Edgar Morin se développe en reliant constamment ce que l'ordre cartésien des raisons avait disjoint. Edgar Morin nous invite à penser «inter et trans disciplinaire » ce qui est également indispensable à l'éducateur qui doit interagir avec des jeunes qui vivent inséparablement, globalement et intensément les différentes dimensions de leur existence.

C'est donc par nécessité de comprendre les relations éducatives que j'ai dû utiliser l'approche systémique dans tous ses développements vers le nouveau paradigme de la pensée complexe, sans la réduire, comme à mes débuts, à la seule systémographie, simple modélisation théorique d'interactions éducatives qui restent abstraites.

\section{PENSER COMPLEXE : NOUVEL HUMANISME ET NOUVELLE RATIONALITÉ}

La critique de la méthode cartésienne analytique classique n'est ici plus à faire : une pensée qui sépare, qui abstrait, et qui réduit (ou avec Aristote, une pensée qui classifie et qui hiérarchise). Au cours des siècles passés, ce grand paradigme de la pensée classique a accumulé les succès, mais toute l'évolution du monde au $\mathrm{XX}^{\mathrm{e}}$ siècle en a montré les effets pervers. Les penseurs les plus innovants ont alors préparé conceptuellement les métamorphoses en cours ou à venir en donnant un visage nouveau à un rationalisme capable d'autocritique et d'ouverture, à la fois conservateur et innovant.

Cependant, une partie de plus en plus importante de la population, ayant subi une éducation encore trop imprégnée des modes de penser cloisonnants, abstrayants, et réducteurs, insécurisée par un mode de vie trop individualiste qui fragmente les groupes sociaux jusqu'à l'atomisation, trouve un nouveau sentiment de communauté par une appartenance forte à des groupes rassurants ayant abandonné toute réflexion critique, toute rationalité, et toute vision globale.

L'exemple épouvantable de la radicalisation djihadiste vient évidemment à l'esprit, mais bien d'autres groupes, beaucoup moins médiatiques parce que beaucoup moins sanglants, sont nés ou se sont transformés en ce sens. On a beaucoup parlé, notamment, des idéologies «new age », et du renouveau des phénomènes sectaires dès les années quatre-vingt. Cependant, d'autres institutions, anciennement fort respectables, connaissent aujourd'hui des dérives totalement irrationnelles, fonctionnant avec des pseudo-théories infalsifiables, cultivant le sophisme et souvent faisant appel à l'archaïsme du bouc émissaire pour souder le groupe. C'est ainsi, par exemple, que l'on a vu récemment des membres de la FNSEA manifester bruyamment pour réclamer le droit de s'empoisonner, d'empoisonner leurs terres ainsi que les consommateurs de leurs productions.

Le nouveau paradigme de complexité nous permet d'échapper à ce « de deux choses l'une » (ou bien l'atomisation techno-scientiste, ou bien le retour à l'obscurantisme et ses confréries fusionnelles). Il s'agit d'une rationalité qui relie. La systémique étudie les interactions, les rétroactions, la communication, tous ces liens à l'intérieur d'un système dont le tout devient plus que la somme des parties. Pour Edgar Morin, le terme central de toute son œuvre qu'il faut retenir et transmettre, c'est la reliance ${ }^{9}$. Ilya Prigogine, lui, a publié en coopération avec

\footnotetext{
${ }^{8}$ Nicolis, G., \& Prigogine, I. (1989, trad. Fr. 1990). A la rencontre du complexe, p.11. Presses Universitaires de France, Paris.

${ }^{9}$ Fert, J.-M. (2012). «Entretien avec Edgar Morin » in Apprendre à penser complexe, vol. II, p. 124. Odin, Paris.
} 
l'épistémologue Isabelle Stengers un essai titré : La Nouvelle alliance ${ }^{10}$. Pour le cybernéticien Von Foerster, en toute rigueur, Descartes n'aurait pas dû écrire : «Je pense donc je suis », mais «Cogito ergo sumus », «je pense donc nous sommes », échappant ainsi au monologue solipsiste $^{11}$.

Dans le domaine éducatif, la mutation nécessaire ne consiste donc en aucun cas à rejeter les savoirs classiques, qu'il s'agisse des sciences ou des « humanités ». Nous voyons dans ce rejet le risque déjà bien actuel d'un retour aux dogmes les plus extrêmes, aux idéologies les plus infalsifiables et, bien loin d'une "société ouverte »" ${ }^{12}$, le retour à une multitude de groupes sectaires clos sur eux-mêmes, imperméables à tout esprit critique et à toute vision globale.

Bien au contraire, l'éducation au penser complexe inclura l'ensemble de ces savoirs dans un ensemble plus vaste et plus ouvert, de la même façon que les théories de Newton sont incorporées comme des cas particulièrement simples dans l'ensemble plus étendu de la relativité. Il conviendra toutefois d'en modifier la pédagogie afin de ne plus les présenter comme des vérités éternelles et absolues (Exemple élémentaire, ne plus dire: "La somme des trois angles d'un triangle est égale à $180^{\circ} »$, sans préciser : «En géométrie euclidienne, la somme ... »). Nous aborderons ces innovations pédagogiques plus précisément dans la suite.

\section{PRÉPARER LA JEUNESSE À UN MONDE EN MUTATION CONSTANTE}

Voici quelques siècles, transmettre les connaissances d'une génération à l'autre était relativement simple, à une époque où les vies humaines étaient courtes, le renouvellement des générations rapides, et les lents changements du monde (notamment les innovations techniques, ainsi que l'organisation politique et religieuse) à peine perceptibles au cours d'une vie. Les lieux d'acquisition de connaissances étaient d'un côté le monastère pour la transmission des textes, de l'autre les compagnons du tour de France pour la transmission des savoir-faire, mais surtout le groupe familial pour la transmission des pratiques locales du «labourage et pâturage ». Les codes de comportements sociaux étaient régis par la religion et la soumission au seigneur. Pour quelques privilégiés, il fallait y ajouter les codes de la chevalerie. Cependant, l'histoire humaine a connu une accélération fulgurante. Rappelons-nous qu'il a fallu plusieurs milliers d'années pour passer de la faucille à la faux, alors que trois siècles plus tard, la faux et les armées de faucheurs étaient remplacées par des énormes moissonneusesbatteuses conduites par un seul homme, et guidées par GPS. Au début de ma carrière, mes formateurs nous prédisaient que la moitié des activités professionnelles que nos élèves auraient à assumer n'existaient pas encore. Ils avaient raison : le téléphone mobile et Internet étaient encore du domaine de la science-fiction.

Nous sommes donc dans une situation totalement inédite où l'éducation, y compris l'éducation parentale, est frappée d'obsolescence si elle se contente d'être la reproduction de celle que les éducateurs ont reçue, car on préparerait alors les jeunes à vivre dans un monde qui n'existe plus, les rendant incapables d'adaptation et d'innovation dans leur monde à venir. Dans notre monde d'hier et d'aujourd'hui, on voyait s'entremêler (et même souvent s'entrechoquer) les questions politiques, sociales et économiques. Dès à présent on voit émerger un monde dans lequel l'intrication est plus étroite que jamais entre les questions psychologiques et religieuses, religieuses et sécuritaires, sécuritaires et démographiques, démographiques et environnementales, environnementales et économiques, économiques et scientifiques, scientifiques et géopolitiques, géopolitiques et psychologiques, et tout cela sans oublier les dimensions existentielles, philosophiques, anthropologiques et éthiques.

\footnotetext{
${ }^{10}$ Prigogine, I. \& Stengers, I. (1979). La Nouvelle alliance. Gallimard, Paris.

${ }^{11}$ Von Foerster, H., op. cit., p. 54.

12 Popper, K. (1945, trad. fr. 1980). La société ouverte et ses ennemis. Seuil, Paris. 
Dans un tel monde, le classicisme aristotélicien ou cartésien devient inopérant, voire nocif car trop réducteur, donc aveugle aux inter rétroactions. Comme le disait Bateson, il faut cesser d'écraser la complexité des situations vécues sur une tautologie unidimensionnelle. Ceux qui tentent encore de donner une explication de phénomènes sociaux complexes à partir d'une seule discipline créent des réalités mutilantes. Aujourd'hui, l'humanité se produit se transmet et se régénère là où les causes sont multiples et enchevêtrées avec les conséquences, là où des idées contraires peuvent être vraies simultanément, là où le tout peut être inclus dans la partie, et là où la liberté humaine émerge malgré d'implacables déterminismes et malgré le chaos aléatoire. L'habitant du monde de demain, acteur-auteur de son exist-ance ${ }^{13}$, doit pouvoir se représenter sa situation et son action en comprenant ces phénomènes d'auto-induction, mais aussi celui de la double contrainte, de la sous-détermination des théories par les faits, de l'auto-organisation à partir du chaos, en comprenant les logiques explosives des relations complémentaires ou symétriques, ainsi que les phénomènes d'inter-rétro-actions entre les paroles et les actes. Il doit aussi inscrire son action et ses innovations dans l'espace social en connaisseur du théorème de Von Foerster-Dupuy. Il lui faudra donc avoir été éduqué à tous les phénomènes mentionnés ci-dessus, de façon à ce qu'il puisse au quotidien s'ouvrir à la complexité du monde, distinguer, comme le proposait déjà Korzybski voici soixante-dix ans, la carte et le territoire (ou le mot et la chose), penser les relations et les processus plutôt que des objets immobiles et non reliés, abandonner la logique classique au profit de logiques postGödeliennes, de dialogiques et de chronologiques, accepter, avec Prigogine, l'irréversibilité du temps qui nous aide à mieux relier la nature et l'homme, s'émerveiller de l'émergence de la complexité plutôt que de la craindre, découvrir et accueillir sa propre complexité, comprendre et accueillir celle des relations sociales, concevoir et accueillir l'ambivalence et la polysémie humaines, comprendre et commencer à exercer sa liberté d'agir, apprendre à verbaliser sa communication, maîtriser les inter-rétro-actions entre la parole et l'acte, afin de devenir auteur de son histoire de vie, et pour finir, plus globalement devenir auteur de son existance.

C'est à travers une telle formation à l'exercice concret d'un «penser complexe » que les nouveaux arrivants dans le monde pourront vivre leur temps comme le propose Edgar Morin dans Les sept savoirs nécessaires à l'éducation du futur ${ }^{14}$ : "La recherche d'un avenir meilleur doit être complémentaire et non plus antagoniste avec les ressourcements dans le passé. Tout être humain, toute collectivité doit irriguer sa vie par une circulation incessante entre son passé où il ressource son identité en se rattachant à ses ascendants, son présent où il affirme ses besoins et un futur où il projette ses aspirations et ses efforts. »C'est à la condition d'une telle formation qu'ils pourront naviguer entre différentes représentations de leur situation, construire leurs réalités au pluriel en évitant les pièges et les effets pervers d'une croyance en l'existence d'une réalité objective unique trop réductrice, et qu'ils pourront donc contribuer à inventer des solutions aux multicrises dont ils héritent, et à mieux vivre dans la complexité du monde actuel et à venir.

\section{L'ÉDUCATION DANS LA PHYLOGENÈSE}

\section{Des soubassements incontournables}

L'éducation peut être définie de multiples façons. Pour moi, il y a paléontologie de l'éducation lorsque l'on trouve des fossiles d'animaux adultes protégeant leurs petits : dès ce moment, voici plusieurs centaines de millions d'années, la complexification du vivant

\footnotetext{
${ }^{13}$ Suivant Derrida, l'emploi d'un « a » rapproche l'existance de l'existant, montrant ainsi qu'il s'agit de quelque chose que l'on fait en participant au présent, et non simplement d'une qualité statique et intemporelle de ce qui est.

${ }^{14}$ Morin, E. (1999). Les sept savoirs nécessaires à l'éducation du futur. Unesco, Paris.
} 
inventait la transmission des acquis d'une génération à la suivante. Dès lors émerge la possibilité d'un héritage autre que génétique, l'héritage de comportements, de l'expérience acquise. L'évolution acquière une nouvelle dimension au-delà du biologique et s'accélère. On aurait tort de réduire cette transmission aux seuls mammifères, puisqu'on sait aujourd'hui que des chants d'oiseaux ne sont pas innés, mais acquis.

La relation mère-enfant est d'une importance littéralement vitale pour un grand nombre d'espèce: les travaux de Boris Cyrulnik ${ }^{15}$ montrent comment on meurt d'un manque d'affection aussi bien chez les humains que chez les animaux. Sur le terrain ${ }^{16}$, c'est l'adoption des petits bonobos par une mère de substitution humaine qui permet leur survie, leur croissance, et ensuite leur retour à la vie dans la forêt. Pour Cyrulnik ${ }^{17}$, c'est au sein de cette chaude relation d'amour maternel que, chez homo sapiens, se fait la naissance du sens.

Nous considérons donc comme acquis le fait que l'éducation précède le langage et conditionne l'accès à l'intellection et à la raison.

Chez les animaux sociaux, le processus connait un nouvel étage de complexité : ce n'est plus seulement les soins maternels par lesquels l'information survit à la succession des générations, mais également par l'apprentissage des rôles et des fonctions à l'intérieur du groupe social. En plus d'être éduqué par sa mère, le petit est aussi éduqué par tous les anciens (dont notamment, pour bon nombre d'espèces, le mâle dominant) ainsi que par ses pairs. On voit alors apparaître une importante complexification et une immense diversification des comportements acquis, dont la plupart ont une fonction de communication et de manifestation concrète des relations sociales au sein du groupe. Selon les espèces, on y développera davantage la communication par les variétés d'attitudes de tout le corps, et/ou de mimiques faciales, et/ou de productions sonores.

C'est ainsi que, dans une importante variété d'espèces, ont pu se développer des comportements sociaux d'une grande complexité et d'une grande valeur de survie collective, tels que le travail d'équipe (notamment dans les techniques de chasse collective des carnivores), la lutte pour la domination (qui n'est pas toujours qu'un combat, mais peut comprendre des phases d'alliances s'apparentant au népotisme et au clientélisme chers à nos édiles), la fraternité (au sens littéral : le soutien à la fratrie dans les conflits ou les épreuves), le tabou de l'inceste (observé au moins entre la mère et le fils chez les bonobos), sans oublier la transmission de savoir-faire techniques (fabrication et maniement d'outils par exemple).

Notons que la richesse, la complexité des interactions entre ces différents modes de communication acquis permet à plusieurs espèces (notamment chez les grands primates) de dépasser le «stade du miroir» théorisé au départ par Henri Wallon comme un moment du développement spécifiquement humain de l'enfant.

Le point important dans ce rappel de la préhistoire naturelle de l'éducation, est de montrer que l'éducation préexiste à l'humanisation, qu'elle ne se réduit pas à une verbalisation de notre connaissance du monde, et notamment que sa fonction de création et transmission du lien social (aujourd'hui pour nous de la «citoyenneté»), ne peut en aucun cas être compris par sa réduction aux seuls apports des neurosciences. Ces nouveaux savoirs sur le fonctionnement du cerveau ont leur validité, mais ne considèrent l'humain qu'individuellement, alors que l'individu (conscient de lui-même) n'émerge dans l'histoire que très récemment, très postérieurement à l'invention du langage, et comme un nouvel étage de l'évolution qui ne

\footnotetext{
${ }^{15}$ Cyrulnik, B. (1983). Mémoire de singe et parole d'homme. Hachette, Paris.

${ }^{16} \mathrm{https}$ ///lolayabonobo.fr/que-faisons-nous/

${ }^{17}$ Cyrulnik, B. (1991). La Naissance du sens. Hachette, Paris. 
saurait se maintenir sans être soutenu par ses soubassements pré-humains : l'amour maternel, l'habileté manuelle (c'est-à-dire déjà une certaine intelligence sensori-motrice) et le lien social.

Justifier des réformes des institutions éducatives par des références aux seules neurosciences relève, comme nous l'avons vu plus haut, de la création de réalités mutilantes.

\section{L'éducation verbale}

Petit à petit, l'éducation maternelle, sociale et technique se faisant, les savoirs s'accumulent donc et se complexifient de génération en génération. Les soins maternels s'améliorent et assurent de meilleures chances de survie des nourrissons, l'habileté manuelle et technique se développe et l'outillage se diversifie et l'ensemble assure une meilleure satisfaction des besoins vitaux, les relations sociales se complexifient également dans des groupes dont l'effectif s'accroit, l'habileté phonatoire se développe également, permettant la communication lorsque les gestes et les mimiques ne peuvent être perçues (la nuit ou à l'intérieur des refuges les plus sombres). Le langage émerge peu à peu, génération après génération, millénaire après millénaire, dans plusieurs groupes de l'espèce «homo » qui n'est pas encore «sapiens». Finalement, la préhistoire devient proprement humaine avec l'invention du «Verbe ». J'ai déjà montré ${ }^{18}$ les liens entre le Verbe, la religion, l'évolution mentale, l'organisation sociale et l'éducation, notamment en me référant aux travaux de Marcel Gauchet ${ }^{19}$ et de Julian Jaynes ${ }^{20}$. C'est toute une histoire de l'évolution de la référence à l'autorité dans l'éducation qui se joue là, depuis le Verbe à l'impératif du Dieu-Roi qui s'impose sous peine de mort, jusqu'à la fameuse phrase de Hannah Arendt, qui écrit dès 1954 que «L'autorité a disparu du monde moderne $»^{21}$. Aujourd'hui, les professeurs et pédagogues sont nombreux à réclamer que ce terme soit banni des discours des ministres successifs, et que l'on officialise les pratiques de terrain basées non sur un impossible retour de l'autorité, mais sur des pédagogies de la confiance.

Par ailleurs, il faut noter une conséquence fondamentale de l'apparition du langage : les êtres humains n'ont pas d'accès conscient (au sens de la conscience verbale réfléchie) au monde sans la médiatisation de la langue, et donc pas d'accès direct au monde mais à la représentation du monde telle que leur langue d'usage le modélise. Je ne pense pas avoir besoin ici d'insister sur cette idée familière aux lecteurs de Watzlawick ${ }^{22}$. Nous voyons apparaître là le risque d'une formation des jeunes qui se réduirait à un conditionnement (ou un endoctrinement, pour reprendre le travail d'Olivier Reboul ${ }^{23}$ ). En effet, si cette formation les éloigne du monde vécu de la sensation, de l'expérience directe, de la confrontation expérientielle, pour les confiner dans un quotidien insipide et répétitif, où la seule nouveauté est celle d'un sur-développement des compétences verbales, l'adhésion sans esprit critique à la «Weltanschauung » enseignée est difficile à éviter. À l'inverse, une formation qui prône un rejet total de toute formation intellectuelle au profit des «bienfaits de l'expérience vécue » risque de limiter la compréhension du monde des jeunes, qui pourraient se mettre à croire que le monde entier fonctionne comme la toute petite partie dont ils ont l'expérience, du fait qu'ils n'ont pas eu l'occasion de s'ouvrir à d'autres modèles. Dans les deux cas, c'est leur capabilité de créativité qui s'en verra catastrophiquement réduite, jusqu'à l'impossibilité de répondre de façon nouvelle et adaptée à des situations nouvelles.

\footnotetext{
${ }^{18}$ Fert, J.-M. (2008). Éduquer pour une société durable - Dieux et autorités en crise. L'Harmattan, Paris.

${ }^{19}$ Gauchet, M. (1985). Le Désenchantement du monde. Gallimard, Paris.

${ }^{20}$ Jaynes, J. (1976, trad. fr. 1994). La Naissance de la conscience dans l'effondrement de l'esprit. P.U.F., Paris.

${ }^{21}$ Arendt, H. (1954, trad. fr. 1972). «Qu'est-ce-que l'autorité ?», in La Crise de la culture. Gallimard, Paris.

${ }^{22}$ Watzlawick, P. \& al. (1981, trad. fr. 1985). L'invention de la réalité, Contributions au constructivisme. Seuil, Paris.

${ }^{23}$ Reboul, O. (1977). L'Endoctrinement. P.U.F., Paris.
} 
Notre approche temporalisée nous a amené jusqu'à la situation récente ou actuelle au cours de laquelle, avec l'apparition de l'écriture, l'éducation prend un nouveau rythme et un nouvel essor.

\section{PÉDAGOGIE DE LA COMPLEXITÉ, PÉDAGOGIE DE LA CRÉATIVITÉ}

\section{Un héritage pesant}

Le temps de l'éducation s'accélère à nouveau, et lorsque l'on invente l'écriture, il ne faut plus que quelques dizaines de siècles pour passer des premières tablettes gravées à l'Internet, et des premières leçons de Socrate à l'apparition des MOOC. Je ne reprendrai pas ici la description de la superposition rapide de différents projets éducatifs qui en France ont fini par générer un mille-feuille institutionnel incohérent et «impilotable $»^{24}$. Rappelons tout de même que si l'École de la République est laïque, elle a gardé de nombreuses traces de son passé catholique, dont notamment ce lancinant appel nostalgique à un retour de l'autorité que nous avons déjà mentionné. Notons également que les deux derniers siècles ont vu naître de grandes institutions dans la plupart des pays dits « développés », par exemple dans le domaine de la santé, de la justice, et bien entendu de l'éducation. La logique du fonctionnement de ces institutions a été critiquée voici presque un demi-siècle par des précurseurs d'une pensée écologisée comme Ivan Illich, qui prédit l'accroissement sans fin de leur coût, le développement de leur contre-productivité, et pour finir leur action corrosive sur l'ensemble de l'organisation sociale ${ }^{25}$. Plus récemment, Philippe Meirieu lui donnait raison en voyant «l'école transformée en machine à désintégrer ${ }^{26}$ alors qu'elle fut créée au contraire pour intégrer la diversité des populations au sein de la République. Mais les effets du «toujours plus de la même chose » sont bien connus des systémiciens et il n'est nul besoin d'insister davantage sur ce point. Pourtant, si l'école-institution devient contre-productive, cela n'empêche pas les éducateurs qui y travaillent de continuer à produire de l'intégration, du lien social, de l'accroissement de compétences, du développement de la confiance en soi, et des capacités créatives chez les jeunes qui leur sont confiés, et tout cela même si c'est au prix de l'accroissement de leur épuisement.

Nous allons donc poursuivre, au-delà de ces logiques instituées dont la dynamique participe de l'aggravation des multi crises évoquées en introduction, par la proposition de visées éducatives qui nous paraissent favorables à la réduction des tensions vécues par les acteurs du terrain éducatif, favorables à la réduction de l'ennui, de la phobie scolaire ou du refus d'apprendre qui sont le quotidien d'un nombre croissant de jeunes, et favorables enfin à l'ouverture de leurs capacités créatrices.

\section{Résistances et reliances : des dynamiques qui se développent}

Nous nous appuierons ici sur les propositions de grands auteurs du siècle dernier et du début de celui-ci. Lorsque j'ai rencontré André de Peretti et Edgar Morin, je leur ai posé la même question: "Quel est le message le plus important que nous devons transmettre à la jeunesse ? ». Pour André de Peretti, il s'agissait de l'esprit de résistance ${ }^{27}$ (et il savait de quoi il parlait, lui qui avait réussi à le maintenir dans les camps nazis de prisonniers) et pour Edgar Morin de la reliance ${ }^{28}$, la nécessité de relier et de se relier.

\footnotetext{
${ }^{24}$ Fert, J.-M. (2014). «Erreurs de représentation dans le domaine de l'éducation » in Teneau, G. (Sous la dir. de), L'Erreur humaine Modèles et représentations. L'Harmattan, Paris.

${ }^{25}$ Illich, I. (1973, trad. fr. 1973). La convivialité. Seuil, Paris.

${ }^{26}$ Meirieu, P. (2011), «L'École transformée en machine à désintégrer », Libération, 9 décembre 2011.

${ }^{27}$ Fert, J.-M. (2008). «Entretien avec André de Peretti », in Éduquer pour une société durable - Dieux et autorités en crise. L'Harmattan, Paris.

${ }^{28}$ Voir plus haut, note 9. 


\section{Résistance éducative et dynamique de projet}

Pour de Peretti, résister, c'est rester soi-même, même dans les circonstances les plus difficiles, ne pas adopter les valeurs de l'adversaire, ne pas se plier au diktat des «de deux choses l'une ", accepter la réalité des circonstances sans s'y soumettre. Lors de la célébration de son centenaire, il nous proposait dix mots ${ }^{29}$ qui éclairent cet esprit de résistance qui fut le sien, et qu'il a inlassablement proposés comme guides pour l'action des éducateurs.

Aujourd'hui, on peut comprendre à partir de cette éthique l'action d'un grand nombre de projets pédagogiques et éducatifs, qu'il s'agisse de projets d'écoles fondées par des groupes de parents et d'enseignants, de mouvements pédagogiques, de projets locaux. Le foisonnement est tel qu'en citer un c'est trahir les autres. Il montre évidemment que, dès que les acteurs sortent de la soumission au carcan institutionnel, dès que l'esprit de résistance hérité de l'engagement d'André de Peretti les anime, ils ne sont plus seulement acteurs de l'éducation, mais deviennent auteurs de projets éducatifs multiformes. La créativité est ici en acte, et bien évidemment, se transmet à la jeunesse à leur contact, car, pour reprendre le mot de Jean Jaurès : «On n'enseigne pas ce que l'on sait ou ce que l'on croit savoir: on n'enseigne et on ne peut enseigner que ce que l'on est. »

Il y a là une tradition de l'insoumission des éducateurs à l'ordre existant qui trouve ses racines dans la vie et la mort de Socrate. En pédagogie ou en éducation, nombreux sont les innovateurs qui, s'ils n'ont pas dû boire la cigüe, ont néanmoins été victimes de calomnies, de mutation d'office, de procès, d'emprisonnement, voire pire. Les biographies des grands noms de l'éducation et de la pédagogie sont toutes parsemées de péripéties douloureuses. En effet les puissances dirigeantes ont toujours préféré former une jeunesse à les servir plutôt qu'à s'émanciper, à défendre l'ordre établi plutôt qu'à innover. C'est dans cette catégorie qu'il faut placer les sophismes et l'inculture à l'œuvre dans les campagnes de calomnies toujours à l'œuvre contre le "pédagogisme $»^{30}$, et qui visent particulièrement la rigueur des travaux considérables de Philippe Meirieu ${ }^{31}$, et plus récemment encore les difficultés que Céline Alvarez ${ }^{32}$ a rencontrées dans l'institution scolaire. On en trouve encore une nouvelle occurrence dans les propos actuels tenus contre les sciences de l'éducation ${ }^{33}$.

Insistons sur un point : nous ne voyons pas dans ce mouvement la promotion d'une pédagogie particulière que l'on pourrait labelliser "pédagogie de la créativité ». On a pu voir, notamment dans les années soixante-dix, des projets soi-disant éducatifs visant à développer la créativité des enfants, en laissant évoluer leur "spontanéité »sans aucun cadre avec du matériel (souvent «artistique ») en libre accès, au nom de leur «bonne nature », avec de fausses références rousseauistes, ou au nom d'une «non-directivité » qui était une terrible trahison des propositions du «Liberté pour apprendre » de Carl Rogers. Pour de Peretti, il ne s'agissait évidemment pas là de résistance, mais d'une simple opposition réactive aux pédagogies autoritaires dominantes. Le « interdit d'interdire » n'a jamais permis de construire quoi que ce soit, et certainement pas un projet éducatif innovant et respectueux des besoins des enfants. Au contraire, il a été utilisé comme prétexte repoussoir par les conservateurs pour contrer toute évolution du système en place.

\footnotetext{
${ }^{29} \mathrm{https}: / / \mathrm{www}$. andredeperetti.net/

${ }^{30}$ Voir la réponse de Philippe Meirieu aux attaques contre les «pédagogues prétentieux » sur le site du Café Pédagogique du 25 novembre 2016 : http://www.cafepedagogique.net/searchcenter/Pages/Results.aspx $? \mathrm{k}=25 \% 20$ novembre\%202016

${ }^{31}$ Meirieu, P. (2017). «À quoi sert la pédagogie ? ", Communication au colloque de l'Institut supérieur de pédagogie de Paris des 28 et 29 mars 2017. https://www.meirieu.com/ARTICLES/ISP_COLLOQUE_PH_MEIRIEU.pdf.

32 Battaglia, M. (2014). «Céline Alvarez, une institutrice révolutionnaire », Le Monde.fr, 4 septembre 2014.

${ }^{33}$ Voir sur http://www.aecse.net le communiqué de presse du conseil d'administration de l'association des enseignants et chercheurs en sciences de l'éducation (AECSE) du 18 octobre 2017.
} 
La rigueur résistante des travaux de Philippe Meirieu, les synthèses qu'il a su opérer des travaux de ses prédécesseurs, nous donnent une base rigoureuse et bien argumentée de réponse à la question fondamentale de toute pédagogie: "Quelles sont les conditions favorables aux processus d'apprentissage ?». Le lecteur pourra se documenter sur le site de Philippe Meirieu, mais il dispose également d'une autre façon de construire sa propre réponse (et ainsi sa propre résistance) : il lui suffit de se remémorer une situation d'acquisition de savoir (ou savoir-faire) agréable et efficace, et une autre à l'opposé. Puis de se rappeler des circonstances personnelles, relationnelles, affectives, instituées... Les évidences arrivent vite. J'ai aussi fait une petite expérience sans aucune rigueur scientifique: j'ai demandé à une dizaine de personnes de me citer, rapidement, sans chercher, une dizaine de choses qu'elles étaient sûres de bien savoir ou de bien savoir-faire, en donnant comme exemple évident « savoir marcher ». Sur la centaine de réponse ainsi collectée, moins d'une dizaine concernait des apprentissages scolaires... On comprend mieux les obstacles auxquels se heurtent les « résistants pédagogiques » qui s'épuisent à rechercher dans le système éducatif, comme le propose André de Peretti, des «Interstices » par lesquels peut se faufiler un changement institutionnel.

\section{Reliances éducatives et dynamique de la confiance}

Pour ce qui est des «reliances » chères à Edgar Morin, je ne développerai pas ici ce point, ne souhaitant pas courir le risque de me répéter, suite à mon article «Reliances en éducation » paru l'an passé ${ }^{34}$. Notons tout de même quelques anciennes évidences, trop fréquemment à l'œuvre dans les situations relationnelles scolaires ici et ailleurs: lorsque l'on se sent en insécurité, lorsque l'on a peur, ce que l'on apprend avant toute autre chose, c'est à vivre dans l'insécurité et dans la peur. Les savoirs au programme, à acquérir, sont secondaires, et ne pourront être utilisées sans réactiver ces «passions tristes », ce que, bien entendu, chacun cherchera à éviter.

La situation relationnelle traditionnellement à l'œuvre fut d'origine religieuse, et se basait sur l'autorité (à la romaine) du maître, laquelle exigeait autant d'obéissance que de soumission chez l'élève, autant d'admiration que de crainte, pour cette figure mâle et âgée qui représentait tout d'abord Dieu, puis la République. Cette tradition s'est estompée, avec la contestation de toutes les anciennes figures de l'autorité, et avec la remise en cause de la domination masculine. Cependant on entend avec un étonnement navré des personnalités publiques en réclamer le retour.

Aujourd'hui, et demain plus encore, la dynamique de la relation éducative est et sera celle de la confiance. L'adulte peut faire confiance à la capacité du jeune à apprendre, le jeune (et ses parents) peuvent faire confiance aux compétences professionnelles de l'éducateur, le jeune peut avoir confiance dans le fait qu'il ne subira pas de conséquence négative s'il se trompe (car seul celui qui sait déjà ne se trompe plus, et n'a donc plus besoin d'apprendre), et les expériences de réussite et d'échec se succédant, la dynamique de la confiance se renforce aussi bien dans la relation à l'autre que dans la relation de chacun à soi-même. De même, non plus seulement dans la relation duelle entre l'éducateur et le jeune, mais dans l'ensemble du groupe, les compétences professionnelles de l'éducateur en matière de pédagogie institutionnelle, sa connaissance des travaux de Fernand Oury, Janusz Korczak, René Lourau et bien d'autres, l'aideront à construire une dynamique de groupe favorable à la progression de tous, et à la résolution des difficultés relationnelles par l'expression verbalisée des conflits, seule prévention réelle de la violence. C'est ainsi au cœur du vécu quotidien que s'inscrit l'acquisition des compétences

\footnotetext{
${ }^{34}$ Fert, J.-M. (2017). «Reliances en éducation» in "Envie d'école", $\mathrm{n}^{\circ}$ 89, journal de la Fédération Nationale des Rééducateurs de l'éducation nationale, publié le 26/01/2017. Consultable sur le site: http://www.editionsharmattan.fr/index.asp?navig=auteurs\&obj=artiste\&no=104 
relatives au «vivre-ensemble », cette convivance si indispensable au développement des capacités collectives de résolution de problèmes.

On entend aujourd'hui encore bon nombre d'idéologue du "libéralisme » survaloriser la «concurrence libre et non faussée ». Tout lecteur de Bateson sait bien que les relations symétriques non régulées mènent à des rétroactions positives et donc à une destruction du système. Les grandes crises économiques sont là pour nous le rappeler, et les régulations par la crise ou par la guerre sont devenues trop coûteuses humainement et écologiquement pour être acceptables. Dans le domaine de l'éducation, il est clair que ces relations entre jeunes doivent impérativement faire place également à des échanges de compétences complémentaires et réciproques. Déjà, dans la Silicon Valley, certaines startup ne veulent plus recruter de polytechnicien car nous disent-ils: "Ils savent tout sauf travailler avec les autres ».

Sur ce point on observe actuellement un grand dynamisme de projets alternatifs, de mise en place de conseils des jeunes, de concertation en «vie de classe » dans les établissements scolaires les plus ordinaires, et bien entendu, c'est aussi le cœur du projet des établissements autogérés ${ }^{35}$.

\section{OUVERTURE FINALE}

Aujourd'hui, les initiatives créatrices en matière de pédagogie et d'éducation sont légion. Elles ne forment pas encore un grand mouvement innovant comme dans l'après 68, mais elles ont appris les leçons tirées de cette époque des «écoles parallèles » et, la plupart du temps, elles ont gagné en rigueur et en compétence. Malheureusement, des projets de petits groupes fermés ayant abandonné tout esprit critique échappent à cette belle dynamique.

Nous avons évoqué ce début de mouvement de transformation-mutation-métamorphose des pratiques et situations éducatives à partir des termes centraux de deux de nos penseurs complexes. Une autre façon d'aborder cette métamorphose serait aussi de montrer que le mouvement en gestation respecte à la fois la conjecture ${ }^{36}$ et l'éthique proposées par Heinz Von Foerster, sur laquelle je vais suspendre mon propos :

- Cogito ergo sumus : Je pense donc nous sommes.

- Toujours s'efforcer d'agir en sorte d'augmenter le nombre des choix possibles; oui, d'augmenter le nombre de choix possibles !

- Si tu veux voir, apprends à agir,

- Si tu veux être toi-même, change $!^{37}$

\footnotetext{
${ }^{35}$ Collectif (2012). Une fabrique de libertés - Le lycée autogéré de Paris. Ed. Repas, Valence.

${ }^{36}$ Voir : Fert, J.-M. (2012). Apprendre à penser complexe, vol. II, pp. 72-78. Odin, Paris.

ou pour un court exposé du théorème de Von Foerster-Dupuy et utilisation dans le champ des métamorphose de l'architecture, le mémoire (pp. 22-25) de ma fille Dounia Fert, consultable et téléchargeable sur le site : http://cargocollective.com/douniafert/ARCHITECTURE/MEMOIRE.

${ }^{37}$ Ces citations sont extraites du document déjà cité. Pour la dernière, elle est à rapprocher de la formulation d'une logique du vivant par Edgar Morin : «Tout ce qui ne se régénère pas dégénère ».
} 
